\title{
Controllability is Not Necessary for Adaptive Pole Placement Control
}

\author{
Han-Fu Chen, Fellow, IEEE, and Xi-Ren Cao, Fellow, IEEE
}

\begin{abstract}
The key issue for adaptive pole-placement control of linear time-invariant systems is the possible singularity of the Sylvester matrix corresponding to the coefficient estimate. However, to overcome the difficulty, the estimate is modified by several methods which are either nonrecursive and with high computational load or recursive but with random search involved. All of the previous works are done under the assumption that the system is controllable. This paper gives the necessary and sufficient condition, which is weaker than controllability, for the system to be adaptively stabilizable. First, a nonrecursive algorithm is proposed to modify the estimates, and the algorithm is proved to terminate in finitely many steps. Then, with the help of stochastic approximation, a recursive algorithm is proposed for obtaining the modification parameters; it is proved that these modification parameters turn out to be a constant vector in a finite number of steps. This leads to the convergence of the modified coefficient estimates. For both algorithms the Sylvester matrices corresponding to the modified coefficient estimates are asymptotically uniformly nonsingular; thus, the adaptive poleplacement control problem can be solved, i.e., the system can be adaptively stabilized.
\end{abstract}

Index Terms-Adaptive pole-placement control, controllability, parameter modification, stochastic approximation.

\section{INTRODUCTION}

$\mathbf{T}$ THE NECESSARY and sufficient condition of arbitrary pole placement for a linear deterministic system with constant coefficients is the controllability of the system. The problem of how to adaptively place poles of a system with unknown coefficients is of practical importance and attracts the attention of many researchers. It seems natural to deal with the adaptive pole-placement problem under the controllability condition. In fact, this is the assumption used in many works in this direction until now. The challenging difficulty is that the Sylvester matrix corresponding to the system with coefficients replaced by their estimates may be degenerate. This prevents one from obtaining the certainty equivalency control which is normally used in adaptive control.

In [1], by using external excitation signals it is proved that the coefficient estimates tend to the true values, and thus the Sylvester matrices corresponding to the coefficient estimates become uniformly nonsingular. This makes it possible to

Manuscript received January 4, 1996; revised March 20, 1997. Recommended by Associate Editor, J. Sun. This work was supported by the National Natural Science Foundation of China, the Hong Kong University of Science and Technology, and the Hong Kong UGC under Grant HKUST 599/94E.

H.-F. Chen is with the Laboratory of Systems and Control, Institute of Systems Science, The Chinese Academy of Sciences, Beijing 100080, China.

X.-R. Cao is with the Department of Electrical and Electronic Engineering, The Hong Kong University of Science and Technology, Clear Water Bay, Kowloon, Hong Kong.

Publisher Item Identifier S 0018-9286(97)06591-4. derive the certainty equivalency control and thus solves the adaptive stabilization problem. The first theoretically complete solution to the problem without invoking external excitation, to the authors' knowledge, belongs to Lozano and Zhao [2], who introduce a coefficient estimation algorithm which is selfconvergent regardless of what control is taken. They choose a modification parameter $\beta_{n}$ to modify the coefficient estimate at each time so that the corresponding Sylvester matrix is uniformly nondegenerate. However, the modification is nonrecursive and bears a very heavy computation burden. For example, for a third-order system each modification requires calculating 46656 determinants of six-dimensional square matrices, and the number of modifications is infinite. Later in [3], the computational load was significantly reduced, and a stopping technique is proposed so that the modification terminates after a finite number of steps. In [4] it is shown that the weighted least squares (WLS) estimate introduced in [5] is self-convergent and has the same convergent rate as the least squares estimate without any modification. Using this estimate, [4] introduces a random search-type algorithm to produce the modification parameter $\beta_{n}$, which guarantees the uniform nondegeneracy of the corresponding Sylvester matrices. However, by the nature of random search the modification procedure must be very slow. All these works were done under the controllability assumption.

The contribution of the present paper consists of the following: 1) The necessary and sufficient condition for the system to be adaptively stabilizable is given. The condition is weaker than controllability, which is well known as the necessary and sufficient condition for nonadaptive exact pole placement. 2) In the proof of the sufficient part a coordinate method for modification is proposed. The method is nonrecursive but the modification terminates in a finite time. 3) Under the same condition a recursive method for modifying estimates is proposed. The modification parameter $\beta_{n}$ equals either zero or $\hat{\beta}_{n}$, which is generated by a stochastic approximation algorithm. Both the modified coefficient estimate and $\left\{\beta_{n}\right\}$ are proved to be convergent. Since stochastic approximation is a directed search algorithm, its convergence rate is hopefully faster than random search. By using modification parameter $\left\{\beta_{n}\right\}$ the adaptive stabilization problem is solved.

\section{THE PROBLEM}

Let us consider the SISO system

$$
A(z) y_{n}=B(z) u_{n}+w_{n}
$$

where $u_{n}, y_{n}$, and $w_{n}$ are the system input, output, and 
disturbance, respectively, and

$$
\begin{aligned}
& A(z)=1+a_{1} z+\cdots+a_{p} z^{p} \\
& B(z)=b_{1} z+\cdots+b_{p} z^{p}
\end{aligned}
$$

where $z$ is the backward shift operator, $z y_{n}=y_{n-1}$.

It is known that the poles of the closed-loop system can arbitrarily be placed if $A(z)$ and $B(z)$ are coprime and if the coefficient

$$
\theta=\left[a_{1}, \cdots, a_{p}, b_{1}, \cdots, b_{p}\right]^{\tau}
$$

is known.

Here we discuss the case where $\theta$ is unknown. Without introducing external excitation signals, an on-line coefficient estimate is hard to keep consistent. Hence, we cannot expect the adaptive pole-placement control to assign desired poles even in asymptotic sense, unless a certain kind of excitation is used [4]. The purpose of adaptive pole-placement control is to achieve

$$
\limsup _{n \rightarrow \infty} \frac{1}{n} \sum_{i=1}^{n}\left(u_{i}^{2}+y_{i}^{2}\right)
$$

System (1) can be written as

$$
y_{n}=\theta^{\tau} \phi_{n-1}+w_{n}
$$

where

$$
\phi_{n-1}^{\tau}=\left[-y_{n-1},-y_{n-2}, \cdots, y_{n-p}, u_{n-1} \cdots u_{n-p}\right] .
$$

Two cases for $w_{n}$ will be considered.

Case 1: $w_{n}$ represents the unmodeled dynamics and satisfies the following condition:

$$
\left|w_{n}\right| \leq \eta+\mu|| \phi_{n-1}||
$$

where $\eta$ and $\mu$ are constants and $\|\cdot\|$ denotes the Euclidean norm.

Case 2: $\left\{w_{n}, \mathcal{F}_{n}\right\}$ is a martingale difference sequence with the following property:

$$
\sup _{n \geq 0} E\left(w_{n+1}^{2} \mid \mathcal{F}_{n}\right)<\infty \quad \text { a.s. }
$$

In what follows, by the estimate $\theta_{n}$ for $\theta$ we mean the one produced by the dead-zone (DZ) algorithm given in [2] for Case 1 and the one generated by the WLS algorithm considered in [4] for Case 2. Due to space limitations, here we only give the WLS algorithm and refer to [2] for the DZ algorithm. The WLS algorithm is defined as follows:

$$
\begin{aligned}
\theta_{n+1} & =\theta_{n}+\frac{P_{n} \phi_{n}}{f\left(r_{n}\right)+\phi_{n}^{\tau} P_{n} \phi_{n}}\left(y_{n+1}-\theta_{n}^{\tau} \phi_{n}\right) \\
P_{n+1} & =P_{n}-\frac{P_{n} \phi_{n} \phi_{n}^{\tau} P_{n}}{f\left(r_{n}\right)+\phi_{n}^{\tau} P_{n} \phi_{n}} \\
r_{n} & =\left\|P_{0}^{-1}\right\|+\sum_{i=0}^{n}\left\|\phi_{i}\right\|^{2}
\end{aligned}
$$

where $f(x)>0$ and $f_{M}^{\infty}(d x / x f(x))<\infty$ for some $M>0$.

It is shown in [2] and [4] that for both DZ and WLS the estimate $\theta_{n}$ and the associated matrix $F_{n}$ are convergent, where $F_{n}=P_{n}^{1 / 2}$ for the WLS algorithm.
It is known [2], [4] that there is a bounded sequence $\left\{\beta_{n}^{0}\right\}$ such that

$$
\theta=\theta_{n}+F_{n} \beta_{n}^{0}
$$

Let us call the following matrix:

$$
M(\theta)=\left[\begin{array}{cccccc}
1 & & 0 & 0 & & 0 \\
a_{1} & \ddots & & b_{1} & \ddots & \\
\vdots & \ddots & 1 & \vdots & \ddots & 0 \\
a_{p} & & a_{1} & b_{p} & & b_{1} \\
& \ddots & \vdots & & \ddots & \vdots \\
0 & & a_{p} & 0 & & b_{p}
\end{array}\right]
$$

the Sylvester matrix corresponding to $\theta$.

It is also known [2], [4] that if a bounded sequence $\left\{\beta_{n}\right\}$ makes the Sylvester matrix corresponding to the modified coefficient estimate

$$
\bar{\theta}_{n}=\theta_{n}+F_{n} \beta_{n}
$$

asymptotically uniformly nondegenerate, i.e.,

$$
\liminf _{n \rightarrow \infty}\left|f\left(\bar{\theta}_{n}\right)\right|>0
$$

where $f(\theta)=\operatorname{det} M(\theta)$, and if $\left\{\bar{\theta}_{n}\right\}$ is convergent, then the control switching between the certainty equivalency control (with $\bar{\theta}_{n}$ used as the coefficient estimate), when $f\left(\bar{\theta}_{n}\right) \neq 0$, and zero, when $f\left(\bar{\theta}_{n}\right)=0$, solves the adaptive pole-placement control problem. The formulation of adaptive control and the proofs of the desired properties of the closed system are given in [2]-[4].

Therefore, the central issue in the adaptive pole-placement control problem is to find a bounded sequence of modification parameters $\left\{\beta_{n}\right\}$ such that $\left\{\bar{\theta}_{n}\right\}$ is convergent and (11) is satisfied. This gives rise to the following definition.

Definition: System (1) is called adaptively stabilizable by the use of the parameter estimate $\theta_{n}$ if there is a bounded sequence $\left\{\beta_{n}\right\}$ such that (11) is satisfied and $\left\{\bar{\theta}_{n}\right\}$ given by (10) is convergent.

Remark 1: From (9) it is seen that if (1) is controllable, then it is adaptively stabilizable by use of $\theta_{n}$, given by the DZ algorithm [2] in Case 1 and by the WLS algorithm [4] in Case 2.

\section{NeCESSARY AND SUfFICIENT CONDITION}

In [2] and [4] it is proved that

$$
\theta_{n} \underset{n \rightarrow \infty}{\longrightarrow} \theta^{\prime}, \quad F_{n} \underset{n \rightarrow \infty}{\longrightarrow} F
$$

where $\theta^{\prime}$, in general, does not equal the true coefficient $\theta$ and may depend on the initial value of (7). Let $\beta$ be $l(=2 p)$ dimensional, $\beta=\left(\beta_{(1)} \cdots \beta_{(l)}\right)^{\tau}$, and consider the polynomial $f\left(\theta^{\prime}+F \beta\right)$ in $\beta$

$$
\begin{aligned}
& f\left(\theta^{\prime}+F \beta\right) \\
& =f\left(\theta^{\prime}\right)+\sum_{i=1}^{l} c_{i}^{(1)} \beta_{(1)}+\sum_{i, j=1}^{l} c_{i, j}^{(2)} \beta_{(i)} \beta_{(j)}+\cdots \\
& \quad+\sum_{i_{1}+\cdots+i_{l}=l} c_{i_{1} \cdots i_{l}}^{(l)} \beta_{(1)}^{i_{1}} \cdots \beta_{(l)}^{i_{l}} .
\end{aligned}
$$


Theorem 1: System (1) is adaptively stabilizable by use of $\theta_{n}$ if and only if

$$
f\left(\theta^{\prime}+F \beta\right) \not \equiv 0
$$

Proof (Necessity): Let (11) be held. Since $\left\{\beta_{n}\right\}$ is bounded, we may select a convergent subsequence $\beta_{n_{k}} \rightarrow \beta^{\prime}$. By (11) and (12) we have $\left|f\left(\theta^{\prime}+F \beta^{\prime}\right)\right|>0$, which verifies (14).

(Sufficiency): Let (14) hold. We now construct a bounded and convergent sequence $\left\{\beta_{n}\right\}$ that satisfies (11). The convergence of $\left\{\bar{\theta}_{n}\right\}$ will be guaranteed by the convergence of $\left\{\beta_{n}\right\}$.

Let $\left\{e_{i}^{(1)}\right\}$ be $l$-dimensional with only one nonzero element equal either to +1 or to $-1, i=1, \cdots, 2 l$. Similarly, let $\left\{e_{i}^{(j)}\right\}$ be $l$-dimensional with only $j$ nonzero elements, each of which equals either 1 or $-1, i=1,2, \cdots,(l(l-1) \cdots(l-j+1) / j !) 2^{j}$.

The total number of such vectors is $L$

$$
L=\sum_{j=1}^{l} \frac{l(l-1) \cdots(l-j+1)}{j !} 2^{j} .
$$

Denote these vectors by $e_{1}, e_{2}, \cdots, e_{L}$ in the nondecreasing order of the number of nonzero elements in $e_{i}$. Before constructing $\bar{\theta}_{n}$ we first make the following observations.

1) If $F=0$, then (14) guarantees $f\left(\theta_{k}\right) \neq 0$ for large $k$, and one may take $\beta_{n} \equiv 0$.

2) If $F \neq 0$ but $f\left(\theta^{\prime}\right) \neq 0$, then one may take $\beta_{n}=0$.

3) We need to construct $\beta_{n}$ only for the case where $F \neq$ $0, f\left(\theta^{\prime}\right)=0$.

It is clear that if $f\left(\theta^{\prime}\right)=0$, then

$$
\max _{i=1 \cdots l}\left|c_{i}^{(1)}\right|=\max _{i}\left[\lim _{\delta \rightarrow 0} \frac{1}{\delta} f\left(\theta^{\prime}+F \delta e_{i}^{(1)}\right)\right] .
$$

If (16) is zero, then

$$
\max _{i, j}\left|c_{i j}^{(2)}\right|=\max _{i}\left[\lim _{\delta \rightarrow 0} \frac{1}{\delta^{2}} f\left(\theta^{\prime}+F \delta e_{i}^{(2)}\right)\right] .
$$

Continuing this procedure, we can finally reach the first nonzero coefficient which is the largest in the sense of absolute value in comparison with other coefficients of the same order; for example, it is achieved at $e_{i}^{(j)}$. Then $\delta e_{i}^{(j)}$, with sufficient small $\delta>0$, may serve as the modification parameter for the remaining time.

We now precisely describe the algorithm.

Take two sequences of real numbers, $\epsilon_{k}>0, \epsilon_{k} \downarrow 0$, $1>\delta_{k}>0, \delta_{k} \downarrow 0$. Set $k=1, \nu_{1}=0$.

1) If $\left|f\left(\theta_{k}\right)\right|>\epsilon_{\nu_{k}}$, then no modification is applied, i.e., $\bar{\theta}_{k}=\theta_{k}$.

2) If $\left|f\left(\theta_{k}\right)\right| \leq \epsilon_{\nu_{k}}$, then from $e_{1}, e_{2}, \cdots e_{L}$ find the smallest integer $k_{0}$ such that

$$
\frac{1}{\delta_{\nu_{k}}^{x(i)}}\left|f\left(\theta_{k}+F_{k} \delta_{\nu_{k}} e_{i}\right)\right| \leq \epsilon_{\nu_{k}}, \quad 1 \leq i \leq k_{0}-1
$$

and

$$
\frac{1}{\delta_{\nu_{k}}^{x(i)}}\left|f\left(\theta_{k}+F_{k} \delta_{\nu_{k}} e_{k_{0}}\right)\right|>\epsilon_{\nu_{k}}
$$

where $x(i)$ is the number of nonzero elements in $e_{i}$.

a) If such a $k_{0} \leq L$ exists, then take $\beta_{k}=\delta_{\nu_{k}} e_{k_{0}}$ and modify $\theta_{k}$ to $\bar{\theta}_{k}=\theta_{k}+F_{k} \delta_{\nu_{k}} e_{k_{0}}$, and use $\bar{\theta}_{k}$ to produce $u_{k}$, which leads to $\theta_{k+1}$; set $\nu_{k+1}=\nu_{k}$. Go back to Step 1).

b) If such a $k_{0}$ does not exist, i.e., (17) holds for all $i=1, \cdots, L$, then set $u_{k}=0$ and calculate $\theta_{k+1}$; set $\nu_{k+1}=\nu_{k}+1$. Go back to Step 1).

We have

$$
\nu_{k}=\sum_{j=1}^{k-1} I_{\left[\left(1 / \delta_{j}^{x(i)}\right)\left|f\left(\theta_{j}+F_{j} \delta_{j} e_{i}\right)\right| \leq \epsilon_{\nu_{j}}, \forall i=1, \cdots, L\right]} .
$$

We now show that under (14), the algorithm defined by 1) and 2) stops at a finite time and $\beta_{k}$ becomes a constant vector with (11) satisfied.

In the case $f\left(\theta^{\prime}\right) \neq 0$, no matter whether $F=0$ or not, the number of transitions from $2 \mathrm{a}$ ) to $2 \mathrm{~b}$ ) can only be finite, i.e., there exists $k_{1}$ such that $\nu_{k}=\nu_{k_{1}}$ and $\beta_{k}=\beta_{k_{1}}, \forall k \geq k_{1}$. This is because $\theta_{k}$ converges to $\theta^{\prime}$ and $\epsilon_{\nu_{k}} \rightarrow 0, \delta_{\nu_{k}} \rightarrow 0$, if $\nu_{k} \rightarrow \infty$. It is clear that one of the following inequalities:

$$
\left|f\left(\theta_{k}\right)\right|>\epsilon_{\nu_{k_{1}}}, \quad\left|f\left(\theta_{k}+F_{k_{k}} \delta_{\nu_{k_{1}}} e_{k_{1}}\right)\right|>\epsilon_{\nu_{k_{1}}}
$$

will hold for all $k \geq k_{1}$. Thus (11) is true.

In the case $f\left(\theta^{\prime}\right)=0$, by (14) not all coefficients of $f\left(\theta^{\prime}+F \beta\right)$ are zero. Therefore, for some $e_{i}^{(j)}$, where $j$ is the lowest nonzero degree, we have

$$
\begin{gathered}
\lim _{\delta \rightarrow 0} \frac{1}{\delta^{j}} f\left(\theta^{\prime}+F \delta e_{i}^{(j)}\right)>0 \\
\left.\max _{i}\left[\lim _{\delta \rightarrow 0} \frac{1}{\delta^{x(s)}} \mid f\left(\theta^{\prime}+F \delta e_{i}^{(s)}\right)\right]\right]=0, \quad 1 \leq s<j .
\end{gathered}
$$

Since $f\left(\theta_{k}+F_{k} \delta e_{i}^{(j)}\right) \underset{k \rightarrow \infty}{\longrightarrow} f\left(\theta^{\prime}+F \delta e_{i}^{(j)}\right)$, there are $\epsilon>0, \Delta>0$, and a sufficiently large $k^{\prime}$ such that

$$
\frac{1}{\delta^{j}}\left|f\left(\theta_{k}+F_{k} \delta e_{i}^{(j)}\right)\right|>\epsilon, \quad \forall k>k^{\prime}, \quad \forall \delta \in[0, \Delta] .
$$

This means that $\nu_{k}$ will not go to infinity, because for sufficiently large $\nu_{k}$, say $\nu_{k_{2}}, \delta_{\nu_{k_{2}}}<\Delta, \epsilon_{\nu_{k_{2}}}<\epsilon$, and (20) implies

$$
\frac{1}{\delta_{\nu_{k_{2}}}^{j}}\left|f\left(\theta_{k}+F_{k} \delta_{\nu_{k_{2}}} e_{i}^{(j)}\right)\right|>\epsilon_{\nu_{k_{2}}}
$$

for all $k>\max \left(k^{\prime}, k_{2}\right)$.

Again, as shown above, once $\nu_{k}$ is finite, $\beta_{k}$ results in being constant and (11) holds.

Remark 2: In the existing adaptive pole-placement control systems [2]-[4], although the system is stabilized, the poles of the homogeneous (i.e., with noise, unmodified dynamics, and reference signal equal to zero) closed-loop system may be asymptotically different from the desired ones if no external excitation signal is introduced. This is because the adaptive pole-placement control is given based on the modified parameter estimate $\bar{\theta}_{n}$, which, in general, is inconsistent. This is the price one has to pay for using the inconsistent estimate to form adaptive control. Since in such an approach one is interested in controllability of (the system corresponding to) $\bar{\theta}_{n}$ [see (11)] and almost all $\theta$ in the $\theta$-space are controllable, intuitively, $\theta_{n}$ can be modified to a controllable $\bar{\theta}_{n}$, regardless 
of whether the open-loop system is controllable or not. This is the reason why controllability is not necessary when forming the adaptive pole-placement control to stabilize the system.

Remark 3: If $F=0$, then $\theta_{n} \rightarrow \theta$, and the polynomial in (14) degenerates to a constant and (14) coincides with controllability of $\theta$. However, $\theta_{n}$, in general, is inconsistent and $F$ is not equal to zero. Therefore, the polynomial in (14) may not degenerate to a constant no matter whether the openloop system (1) is controllable or not. This explains why (14) is weaker than controllability.

Remark 4: In the proof of Theorem 1 under (14), a coordinate method is used to design the algorithm for modifying coefficient estimates. The modification terminates in a finite number of steps. This greatly saves the computation and makes the algorithm practically implementable.

\section{Recursive Method for Constructing $\left\{\beta_{n}\right\}$}

In this section, using a stochastic approximation algorithm we recursively produce the modification parameter $\beta_{n}$ in (10) which is convergent and satisfies (11).

We first introduce a stochastic approximation algorithm seeking nonzero roots of the function

$$
\begin{aligned}
h(\beta) \triangleq & -\left(\frac{\partial f\left(\theta^{\prime}+F \beta\right)}{\partial \beta}-l\|\beta\|^{l-2} \beta\right) \\
& \cdot\left(f\left(\theta^{\prime}+F \beta\right)-\|\beta\|^{l}-b\right), \quad b>0 .
\end{aligned}
$$

As a matter of fact, if we set

$$
v(\beta)=\left(f\left(\theta^{\prime}+F \beta\right)-\|\beta\|^{l}-b\right)^{2}
$$

then

$$
h(\beta)=-\frac{1}{2} \frac{\partial v(\beta)}{\partial \beta} .
$$

Let us denote the root set of (21) by $J=\{\beta: h(\beta)=0\}=$ $J_{1} \cup J_{2}$, where

$$
\begin{aligned}
& J_{1}=\left\{\beta: \frac{\partial f\left(\theta^{\prime}+F \beta\right)}{\partial \beta}-l\|\beta\|^{l-2} \beta=0\right\} \\
& J_{2}=\left\{\beta: f\left(\theta^{\prime}+F \beta\right)-\|\beta\|^{l}-b=0\right\} .
\end{aligned}
$$

However, $h(\beta)$ cannot be directly observed; the real observation is

$$
\begin{aligned}
y_{k+1}= & -\left(\frac{\partial f\left(\theta_{k}+F_{k} \hat{\beta}_{k}\right)}{\partial \beta}-l\left\|\hat{\beta}_{k}\right\|^{l-2} \hat{\beta}_{k}\right) \\
& \cdot\left(f\left(\theta_{k}+F_{k} \hat{\beta}_{k}\right)-\left\|\hat{\beta}_{k}\right\|^{l}-b\right)
\end{aligned}
$$

which can be written as

$$
y_{k+1}=h\left(\hat{\beta}_{k}\right)+\xi_{k+1}
$$

where $\xi_{k+1}=y_{k+1}-h\left(\hat{\beta}_{k}\right)$, and $\hat{\beta}_{k}$ is recursively generated by (28).

For defining the algorithm for $\hat{\beta}_{k}$, let us take a few real sequences defined as follows:

$$
\begin{aligned}
M_{k} & >0, \quad M_{k} \uparrow \infty, \quad \epsilon_{k}>0, \quad \epsilon_{1}<\frac{1}{2}, \quad \epsilon_{k} \downarrow 0 \\
\delta_{k} & >0, \quad \delta_{k} \downarrow 0, \quad \delta_{1}<\frac{1}{2} .
\end{aligned}
$$

In addition, we take the sequence of stepsizes $\left\{a_{k}\right\}, k=$ $1,2, \cdots$.

Let us normalize vectors $e_{1}, \cdots, e_{L}$ and denote the resulting vectors by $e_{1}^{0}, \cdots, e_{L}^{0}$. Define $e_{i}^{*}=e_{i}^{0}, i=1, \cdots, L$, and $e_{i}^{*}=e_{i-[i / L] L}^{0}$ for $i>L$.

Introduce

$$
v_{k}(\beta)=\left[f\left(\theta_{k}+F_{k} \beta\right)-\|\beta\|^{l}-b\right]^{2} .
$$

Define the recursive algorithm for $\left\{\hat{\beta}_{k}\right\}$ as follows:

$$
\begin{aligned}
\bar{\beta}_{k+1}= & \hat{\beta}_{k}+a_{k} y_{k+1} \\
\hat{\beta}_{k+1}= & \bar{\beta}_{k+1} I_{\left[\left\|\bar{\beta}_{k+1}\right\| \leq M_{\sigma_{k}}\right] \cap\left[\left\|\bar{\beta}_{k+1}\right\| \geq \epsilon_{\tau_{k}}\right]} \\
& +\beta^{*} I_{\left[\left\|\bar{\beta}_{k+1}\right\|>M_{\sigma_{k}}\right]}+\delta_{\mu_{k}} e_{\gamma_{k}}^{*} I_{\left[\left\|\bar{\beta}_{k+1}\right\|<\epsilon_{\tau_{k}}\right]} \\
\beta_{0} \gg & \epsilon_{1}, \quad \operatorname{say} \hat{\beta}_{0}=10 \\
\sigma_{n}= & \sum_{k=1}^{n-1} I_{\left[\left\|\bar{\beta}_{k+1}\right\|>M_{\sigma_{k}}\right]}, \quad \tau_{n}=\sum_{k=1}^{n-1} I_{\left[\left\|\bar{\beta}_{k+1}\right\|<\epsilon_{\tau_{k}}\right]} \\
\gamma_{n}= & \sum_{k=1}^{n-1} I_{\left[v_{k+1}\left(\delta_{\mu_{k}} e_{\gamma_{k}}^{*}\right) \geq b^{2}\right]}^{n-1} I_{\left[\tau_{k}-\tau_{k-L}=\gamma_{k}-\gamma_{k-L}\right]} \\
\mu_{n}= & \sum_{k=L+1}
\end{aligned}
$$

and $\beta^{*}$ is a fixed vector, $\left\|\beta^{*}\right\|>1$.

Here we explain the meaning of the algorithm (27)-(30). The algorithm basically is the Robbins-Monro (RM) algorithm truncated at randomly varying bounds. From the upper side, at time $k$ it is truncated at $M_{\sigma_{k}}$, and after each truncation the algorithm is pulled back to $\beta^{*}$ and the truncation bound enlarged from $M_{\sigma_{k}}$ to $M_{\sigma_{k}+1}$. At the lower side we do not allow $\beta_{k}$ to tend to zero. Each time it reaches the lower bound $\epsilon_{\tau_{k}}$ we pull it back to $e_{\gamma_{k}}^{*}$, which will change to the next $e_{\gamma_{k}+1}^{*}$ whenever $\left[v_{k+1}\left(\delta_{\mu_{k}} e_{\gamma_{k}}^{*}\right) \geq b^{2}\right]$ is satisfied. If for $L$ successive resettings of $e_{\gamma_{k}}^{*}$ we have to change $e_{\gamma_{k}}^{*}$ to the next one, then we reduce $\delta_{\mu_{k}}$ to $\delta_{\mu_{k}+1}$.

Theorem 2: Assume the stepsize satisfies the following conditions:

$$
a_{k}>0, \quad a_{k} \rightarrow 0, \quad \sum_{k=1}^{\infty} a_{k}=\infty .
$$

If (14) holds and $f\left(\theta^{\prime}\right)=0$, then:

1) after a finite number of steps the algorithm (27)-(30) becomes the RM algorithm

$$
\hat{\beta}_{k+1}=\hat{\beta}_{k}+a_{k} y_{k+1}
$$

2) $v\left(\hat{\beta}_{n}\right)$ converges;

3) $\lim _{n \rightarrow \infty} d\left(\hat{\beta}_{n}, J \backslash\{0\}\right)=0$.

We now describe the algorithm recursively constructing the modification parameters $\left\{\beta_{n}\right\}$.

Let $\eta_{k}>0, \eta_{k} \downarrow 0$.

1) If $\left|f\left(\theta_{k}\right)\right|>\eta_{\lambda_{k}}$, then set $\beta_{k}=0$, i.e., $\bar{\theta}_{k}=\theta_{k}$. Using $\theta_{k}$ we produce $u_{k}$ and go back to 1) for $k+1$.

2) If $\left|f\left(\theta_{k}\right)\right| \leq \eta_{\lambda_{k}}$, then define

a) $\beta_{k}=\beta_{k-1}$ for the case where $\mid f\left(\theta_{k}+\right.$ $F_{k}\left(\beta_{k-1}\right) \mid>\eta_{\lambda_{k}}$ 
b) $\beta_{k}=\hat{\beta}_{k}$ defined by (27)-(30) for the case where

$\left|f\left(\theta_{k}+F_{k} \beta_{k-1}\right)\right| \leq \eta_{\lambda_{k}}, \quad$ but $\left|f\left(\theta_{k}+F_{k} \hat{\beta}_{k}\right)\right|>\eta_{\lambda_{k}} ;$

c) $\beta_{k}=\left(1+\sqrt{\eta_{\lambda_{k}}}\right) \hat{\beta}_{k}$ for the case, where

$\left|f\left(\theta_{k}+F_{k} \beta_{k-1}\right)\right| \leq \eta_{\lambda_{k}}, \quad\left|f\left(\theta_{k}+F_{k} \hat{\beta}_{k}\right)\right| \leq \eta_{\lambda_{k}}$

but

$$
\left|f\left(\theta_{k}+F_{k}\left(1+\sqrt{\eta_{\lambda_{k}}}\right) \hat{\beta}_{k}\right)\right|>\eta_{\lambda_{k}} .
$$

Define

$$
\bar{\theta}_{k}=\theta_{k}+F_{k} \beta_{k}
$$

and use $\bar{\theta}_{k}$ to produce $u_{k}$ and go back to 1 ) for $k+1$.

3) If $\left|f\left(\theta_{k}\right)\right| \leq \eta_{\lambda_{k}}$ and none of a)-c) of 2) is the case, then set $\beta_{k}=0$ and $u_{k}=0$, go back to 1$)$ for $k+1$, and at the same time change $\eta_{\lambda_{k}}$ to $\eta_{\lambda_{k}+1}$, i.e.,

$$
\lambda_{k}=\sum_{i=1}^{k-1} I_{\left[\left|f\left(\theta_{i}\right)\right| \leq \eta_{\lambda_{i}} ;\left|f\left(\theta_{i}+F_{i} \beta_{i-1}\right)\right| \leq \eta_{\lambda_{i}} ;\right.}^{\left.\left|f\left(\theta_{i}+F_{i} \hat{\beta}_{i}\right)\right| \leq \eta_{\lambda_{i}} ;\left|f\left(\theta_{i}+\left(1+\sqrt{\eta \lambda_{i}}\right) F_{i} \hat{\beta}_{i}\right)\right| \leq \eta_{\lambda_{i}}\right]}
$$

where $\bar{\theta}_{k}$ is defined by (33).

Let us call 1)-3) $\left\{\beta_{n}\right\}$ for defining the modification algorithm.

Theorem 3: Suppose (31) and (14) hold. Then there is $k_{0}$ such that $\beta_{k} \equiv \beta_{k_{0}} \forall k \geq k_{0},\left\{\bar{\theta}_{k}\right\}$ defined by (33) converges and

$$
\liminf _{k \rightarrow \infty}\left|f\left(\bar{\theta}_{k}\right)\right|>0
$$

\section{Proof of Theorems 2 AND 3}

We start with the lemmas. Define

$$
m(n, T)=\max \left\{m: \sum_{i=n}^{m} a_{i} \leq T\right\}, \quad T>0 .
$$

Lemma 1: Let $\left\{\hat{\beta}_{n_{k}}\right\}$ be a convergent subsequence of $\left\{\hat{\beta}_{n}\right\}, \hat{\beta}_{n_{k}} \underset{k \rightarrow \infty}{\longrightarrow} \beta^{\prime}$. Starting from any $n_{k}$ define the RM algorithm

$$
\begin{aligned}
\beta_{m+1}^{\prime}= & \beta_{m}^{\prime}+a_{m} y_{m+1}^{\prime} \\
\beta_{n_{k}}^{\prime}= & \hat{\beta}_{n_{k}} \text { or } \beta_{n_{k}}^{\prime}=\delta_{\mu_{j}} e_{\gamma_{j}}^{*} \text { for some } j \\
y_{m+1}^{\prime}= & -\left(f\left(\theta_{m}-F_{m} \beta_{m}^{\prime}\right)-\left\|\beta_{m}^{\prime}\right\|^{l}-b\right) \\
& \cdot\left(\frac{\partial f\left(\theta_{m}+F_{m} \beta_{m}^{\prime}\right)}{\partial \beta}-l\left\|\beta_{m}^{\prime}\right\|^{l-2} \beta_{m}^{\prime}\right) .
\end{aligned}
$$

If (31) holds, then there are $M>0, T>0$ such that

$$
\left\|\beta_{m}^{\prime}\right\| \leq M, \quad \forall m \in\left\{n_{k}, \cdots, m\left(n_{k}, T\right)\right\}, \quad \forall k \geq k_{0}
$$

if $k_{0}$ is large enough. (In what follows, $k_{0}$ always denotes a sufficiently large integer; it may vary from time to time.)
Proof: Let $c>1$ be a constant such that

$$
\left\|\beta_{n_{k}}^{\prime}\right\|<c, \quad \forall k \geq 1 .
$$

Since $\theta_{k}$ and $F_{k}$ are convergent, there is $\alpha<\infty$ such that

$$
\begin{aligned}
\max _{\substack{\|\beta\| \leq 2 c \\
1 \leq k<\infty}}\left|f\left(\theta_{k}+F_{k} \beta\right)-\|\beta\|^{l}-b\right| \\
\quad \cdot\left\|\frac{\partial f\left(\theta_{k}+F_{k} \beta\right)}{\partial \beta}-l\right\| \beta\left\|^{l-2} \beta\right\|=\alpha<\infty .
\end{aligned}
$$

Let $T<(c / 2 \alpha)$. We show that we can take

$$
M=\frac{3 c}{2} .
$$

By (35), (34) is true for $n_{k}$. Let (34) hold for $i=n_{k}, n_{k}+$ $1, \cdots, m<m\left(n_{k}, T\right)$. Then we have

$$
\begin{gathered}
\left\|\beta_{m+1}^{\prime}\right\| \leq\left\|\beta_{n_{k}}^{\prime}\right\|+\sum_{i=n_{k}}^{m} a_{i}\left|f\left(\theta_{i}+F_{i} \beta_{i}^{\prime}\right)-\left\|\beta_{i}^{\prime}\right\|^{l}-b\right| \\
\cdot\left\|\frac{\partial f\left(\theta_{i}+F_{i} \beta_{i}^{\prime}\right)}{\partial \beta}-l\right\| \beta_{i}^{\prime}\left\|^{l-2} \beta_{i}^{\prime}\right\| \\
<c+\sum_{i=n_{k}}^{m} a_{i} \alpha \leq c+T \alpha<\frac{3 c}{2} .
\end{gathered}
$$

Thus, (34) has been inductively proved.

Lemma 2: Let (31) hold and let $\left\{\hat{\beta}_{n_{k}}\right\}$ be a convergent subsequence. There are $M>0, T>0$ such that

$$
\left\|\hat{\beta}_{m}\right\| \leq M, \quad \forall m \in\left\{n_{k}, \cdots, m\left(n_{k}, T\right)\right\}, \quad \forall k \geq k_{0}
$$

if $k_{0}$ is sufficiently large.

Proof: If $\sigma_{n}$ defined by (29) is bounded, then (36) directly follows.

Again take $c>1$ such that $\left\|\hat{\beta}_{n_{k}}\right\|<c, \forall k \geq 1$ and set $M=3 c / 2$.

Assume $\sigma_{n} \rightarrow \infty$. Then there is a $k_{0}$ such that

$$
M_{\sigma_{k_{0}}}>M \text {. }
$$

By Lemma 1, starting from $\hat{\beta}_{n_{k}}, k \geq k_{0}$, the algorithm for $\hat{\beta}_{i}$ cannot directly hit the sphere with radius $M_{\sigma_{k_{0}}}$ without a truncation for $i \in\left\{n_{k}, n_{k}+1, \cdots, m(n, T)\right\}$. So it may first hit some lower truncation bound and switch to some $\delta_{\mu_{j}} e_{\gamma_{j}}^{*}$, from which again by Lemma $1 \hat{\beta}_{i}$ cannot directly reach $M_{\sigma_{k_{0}}}$ without a truncation. The only possibility is to be truncated again at a lower bound. Inductively using this argument proves (36).

Note that $f\left(\theta^{\prime}+F \beta\right)$ is a polynomial in $\beta_{(i)}, i=1, \cdots, l$, and its highest possible degree is $l-1$. Both polynomials $f\left(\theta^{\prime}+F \beta\right)-\|\beta\|^{l}-b$ and $\left(\partial f\left(\theta^{\prime}+F \beta\right) / \partial \beta\right)-l\|\beta\|^{l-1} \beta$ diverge to infinity as $\|\beta\| \rightarrow \infty$. Therefore, $J$ is a bounded set. Clearly, it is also closed. 
Lemma 3: $v(J)$ is a finite set, where

$$
v(J)=\{v(\beta): \beta \in J\}
$$

This is a fact from algebraic geometry. For the proof we refer to [6].

Since $v(\beta) \rightarrow \infty$ as $\|\beta\| \rightarrow \infty$, there is $c_{0}$ such that

$$
\left\|\beta^{*}\right\|<c_{0}, \quad v\left(\beta^{*}\right)<\inf _{\|\beta\|=c_{0}} v(\beta)
$$

and by Lemma 3

$$
(v(J))^{c} \cap\left(v\left(\beta^{*}\right), \quad \inf _{\|\beta\|=c_{0}} v(\beta)\right) \neq \emptyset
$$

By using Lemma 2 and the convergence of $\theta_{k}$ and $F_{k}$, it is clear that for any convergent subsequence $\left\{\hat{\beta}_{n_{k}}\right\}$ we have

$$
\lim _{T \rightarrow 0} \lim _{k \rightarrow \infty} \frac{1}{T} \sum_{i=n_{k}}^{m\left(n_{k}, T\right)} a_{i} \xi_{i+1}=0
$$

In the sequel, we will say that the sequence $v\left(\hat{\beta}_{n_{k}}\right) \cdots v\left(\hat{\beta}_{m_{k}}\right)$ crosses an interval $\left[\delta_{1}, \delta_{2}\right]$ if $v\left(\hat{\beta}_{n_{k}}\right) \leq$ $\delta_{1}, v\left(\hat{\beta}_{m_{k}}\right) \geq \delta_{2}$ and $\delta_{1}<v\left(\hat{\beta}_{i}\right)<\delta_{2}, \forall i ; n_{k}<i<m_{k}$.

$\hat{\beta}_{n_{k}}$ always denotes the starting point of a crossing, $k=$ $1,2, \cdots$.

Lemma 4: Let (31) hold. Assume that starting from $\hat{\beta}_{n_{k}}, k=1,2, \cdots$, algorithm (28) is calculated as RM and $\left\{\| \hat{\beta}_{n_{k}}||, k=1,2, \cdots\right\}$ is bounded. Then for any $\left[\delta_{1}, \delta_{2}\right]$ with $d\left(\left[\delta_{1}, \delta_{2}\right], v(J)\right)>0, v\left(\hat{\beta}_{n}\right)$ cannot cross $\left[\delta_{1}, \delta_{2}\right]$ i.o., if $\delta_{2}>\delta_{1}$ or $v\left(\hat{\beta}_{n}\right)$ cannot tend to $\delta_{1}$ if $\delta_{1}=\delta_{2}$.

For the proof we refer to [7] and [8], where (39) and (40) are the required conditions. The lower bound truncation used in (28) is not a problem because we have assumed that the algorithm develops as an RM starting from $\hat{\beta}_{n_{k}}$.

Lemma 5: Assume (31) holds. Then the sequence $\left\{\hat{\beta}_{n}\right\}$ defined by (28) is bounded.

Proof: If $\left\{\hat{\beta}_{n}\right\}$ is unbounded, then $\sigma_{n} \underset{n \rightarrow \infty}{\longrightarrow} \infty$. Therefore, $\left\{v\left(\hat{\beta}_{n}\right)\right\}$ is unbounded and comes back to the fixed point $v\left(\beta^{*}\right)$ infinitely many times. Since $v\left(\delta_{\mu_{k}} e_{\gamma_{k}}^{*}\right) \leq$ $\max _{\|\beta\| \leq(1 / 2)} v(\beta) \triangleq \zeta<\infty$, by Lemma 3 there is an interval $\left[\delta_{1}, \delta_{2}\right]$ with $\delta_{1}>\zeta$ and $d\left(\left[\delta_{1}, \delta_{2}\right], v(J)\right)>0$ such that $v\left(\hat{\beta}_{n}\right)$ crosses $\left[\delta_{1}, \delta_{2}\right]$, i.o., and starting from $\hat{\beta}_{n_{k}}$ the algorithm (28) behaves like an RM while $v\left(\hat{\beta}_{n}\right)$ crosses $\left[\delta_{1}, \delta_{2}\right]$. It is clear that $\left\{\hat{\beta}_{n_{k}}\right\}$ is bounded because $v(\beta) \rightarrow \infty$ as $\|\beta\| \rightarrow \infty$. By Lemma 4 this is impossible. Thus, we conclude that $\left\{\hat{\beta}_{n}\right\}$ is bounded.

Remark 5: Lemmas 1-5 are proved without using (14) and $f\left(\theta^{\prime}\right)=0$. After a finite number of steps, (28) becomes

$$
\hat{\beta}_{k+1}=\bar{\beta}_{k+1} I_{\left[\left\|\bar{\beta}_{k+1}\right\| \geq \epsilon_{\tau_{k}}\right]}+\delta_{\mu_{k}} e_{\gamma_{k}}^{*} I_{\left[\left\|\bar{\beta}_{k+1}\right\|<\epsilon_{\tau_{k}}\right]} .
$$

Lemma 6: If (31) and (14) hold and $f\left(\theta^{\prime}\right)=0$, then there exists a $k_{0}$ such that the recursive equation (41) for $\left\{\hat{\beta}_{k}\right\}$ becomes an RM algorithm (32) for $k \geq k_{0}$.
Proof: From (19) it is seen that for some $e_{i}^{(j)}$ and a small $\epsilon>0$

$$
f\left(\theta^{\prime}+F \epsilon e_{i}^{(j)}\right)>0
$$

which tends to zero as $\epsilon \rightarrow 0$.

Noticing that the highest degree of $f\left(\theta^{\prime}+F \beta\right)$ is less than or equal to $l-1$, for sufficiently small $\epsilon>0$, we have

$$
v\left(\epsilon e_{i}^{(j)}\right)<b^{2}=v(0) .
$$

From this and convergence of $\theta_{k}$ and $F_{k}$, it follows that

$$
v_{k}\left(\epsilon e_{i}^{(j)}\right)<b^{2}
$$

for all sufficiently large $k$. Therefore, $\left\{\gamma_{n}\right\}$ and hence $\left\{\mu_{k}\right\}$ must be bounded, i.e., there is a $k_{0}$ such that

$$
\delta_{\mu_{k}}=\delta_{\mu_{k_{0}}}, \quad e_{\gamma_{k}}^{*}=e_{\gamma_{k_{0}}}^{*}, \quad \forall k \geq k_{0}
$$

We now show that $\left\{\tau_{n}\right\}$ is bounded. Assume the converse is true. We have

$$
v_{k}\left(\delta_{\mu_{k_{0}}} e_{\gamma_{k_{0}}}^{*}\right)<b^{2}, \quad \text { for all sufficiently large } k
$$

and hence

$$
v\left(\delta_{\mu_{k_{0}}} e_{\gamma_{k_{0}}}^{*}\right) \triangleq a<b^{2}
$$

The algorithm, starting from $\delta_{\mu_{k_{0}}} e_{\gamma_{k_{0}}}^{*}$, infinitely many times enters the sphere with radius $\epsilon:\{\beta:\|\beta\|<\epsilon\}$, where $\epsilon$ is small enough such that

$$
\min _{\|\beta\| \leq \epsilon} v(\beta)=a+\delta \leq b^{2}, \quad \delta>0 .
$$

The existence of such an $\epsilon$ is guaranteed because

$$
v(\beta) \rightarrow b^{2} \text { as } \beta \rightarrow 0 .
$$

Then $v\left(\hat{\beta}_{n}\right)$ would cross infinitely often an interval $\left[\delta_{1}, \delta_{2}\right] \in$ $(a, a+\delta)$. By Lemma 3, we may assume $d\left(\left[\delta_{1}, \delta_{2}\right], v(J)\right)>0$. It is clear that during the crossing the algorithm behaves like an RM. By Lemma 4, this is impossible.

Proof of Theorem 2: By Lemma 6, there is a $k_{0}$ such that

$$
\hat{\beta}_{k+1}=\hat{\beta}_{k}+a_{k} y_{k+1}, \quad \forall k \geq k_{0}
$$

and $\hat{\beta}_{k}$ cannot tend to zero.

Assume

$$
v_{1}=\liminf _{n \rightarrow \infty} v\left(\hat{\beta}_{n}\right) \leq \limsup _{n \rightarrow \infty} v\left(\hat{\beta}_{n}\right)=v_{2} .
$$

If $v_{2}>v_{1}$, then by Lemma 3 there is an interval $\left[\delta_{1}, \delta_{2}\right] \subset$ $\left[v_{1}, v_{2}\right]$ such that $\left[\delta_{1}, \delta_{2}\right] \cap v(J)=\emptyset$ and $v\left(\beta_{n}\right)$ crosses $\left[\delta_{1}, \delta_{2}\right]$ i.o. By Lemma 4 this is impossible. Therefore $v_{1}=v_{2}$, and $v\left(\hat{\beta}_{n}\right)$ converges. Again by Lemma 4 , in this case $v_{1}=v_{2} \in$ $v(J)$.

Let $\beta^{0}$ be a limit point of $\left\{\hat{\beta}_{n}\right\}$, i.e., there is a subsequence $\left\{\hat{\beta}_{n_{k}}\right\}$ such that $\hat{\beta}_{n_{k}} \underset{k \rightarrow \infty}{\longrightarrow} \beta^{0}$. Assume the converse $\beta^{0} \bar{\epsilon} J$. Then

$$
\left(\frac{\partial v\left(\beta^{0}\right)}{\partial \beta}\right)^{\tau} h\left(\beta^{0}\right)=-\frac{1}{2}\left\|\frac{\partial v\left(\beta^{0}\right)}{\partial \beta}\right\| \triangleq-a<0 .
$$


For $n_{k} \geq k_{0}$ with $k_{0}$ given by (42), by the Taylor expansion we have

$$
v\left(\hat{\beta}_{m\left(n_{k}, T\right)+1}\right)-v\left(\hat{\beta}_{n_{k}}\right)=\left(\frac{\partial v(\hat{\beta})}{\partial \beta}\right)^{\tau} \sum_{i=n_{k}}^{m\left(n_{k}, T\right)} a_{i} y_{i+1}
$$

where $\hat{\beta}$ 's components are in between the corresponding components of $\hat{\beta}_{m\left(n_{k}, T\right)+1}$ and $v\left(\hat{\beta}_{n_{k}}\right)$. From here it follows that

$$
\begin{aligned}
& v\left(\hat{\beta}_{m\left(n_{k}, T\right)+1}\right)-v\left(\hat{\beta}_{n_{k}}\right) \\
& \quad=\sum_{i=n_{k}}^{m\left(n_{k}, T\right)} a_{i}\left(\frac{\partial v\left(\beta^{0}\right)}{\partial \beta}\right)^{\tau} h\left(\beta^{0}\right)+o(T)
\end{aligned}
$$

where $o(T) \rightarrow 0$ as $T \rightarrow 0$ uniformly in $k$. Tending $k \rightarrow \infty$, the left-hand side of (43) tends to zero, while the right-hand side converges to $-a T+o(T)$. The contradiction means that $\beta^{0} \in J$. By Lemma $6 \beta^{0}$ cannot be zero.

Since $\beta^{0}$ is an arbitrary limit point, we conclude $\lim _{n \rightarrow \infty} d\left(\hat{\beta}_{n}, J \backslash\{0\}\right)=0$.

Proof of Theorem 3: We first show that for the proof it suffices to show $\lim _{k \rightarrow \infty} \lambda_{k}<\infty$.

If $\lim _{k \rightarrow \infty} \lambda_{k}<\infty$, then there is a $k_{0}$ such that for $k \geq$ $k_{0} \lambda_{k} \equiv \lambda_{k_{0}}$ the modification algorithm will run over the following cases: 1) and 2a)-2c). Since $\theta_{k}$ and $F_{k}$ are convergent, the inequality $\left|F\left(\theta_{k}+F_{k} \hat{\beta}_{k}\right)\right|>\eta_{\lambda_{k_{0}}}$ implies $\mid F\left(\theta_{k+i}+\right.$ $\left.F_{k+i} \hat{\beta}_{k}\right) \mid>\eta_{\lambda_{k_{0}}}, \forall i \geq 1$ for sufficiently large $k$. This means that the modification algorithm can be at $2 \mathrm{~b}$ ) only for finitely many times. By the same reason, it cannot be at 2c) for infinitely many times. Therefore, the algorithm will stick on 1) if $\left|f\left(\theta^{\prime}\right)\right|>\eta_{\lambda_{k_{0}}}$ and on 2b) if $\left|f\left(\theta^{\prime}\right)\right| \leq \eta_{\lambda_{k_{0}}}$, and in both cases there is a $k_{0}$ such that $\beta_{k}=\beta_{k_{0}}, \forall k \geq k_{0}$ and

$$
\liminf _{k \rightarrow \infty}\left|f\left(\bar{\theta}_{k}\right)\right|>\eta_{\lambda_{k_{0}}}>0 .
$$

The convergence of $\bar{\theta}_{k}$ follows from the convergence of $\theta_{k}$ and $F_{k}$.

We now show that $\lim _{k \rightarrow \infty} \lambda_{k}<\infty$.

Assume the converse: $\lambda_{k} \underset{k \rightarrow \infty}{\longrightarrow} \infty$.

Case a $\left(\left|f\left(\theta^{\prime}\right)\right| \neq 0\right)$ : The assumption $\lambda_{k} \underset{k \rightarrow \infty}{\longrightarrow} \infty$ implies that $\eta_{\lambda_{k}} \underset{k \rightarrow \infty}{\longrightarrow} 0$ and $\left|f\left(\theta_{k}\right)\right| \leq \eta_{\lambda_{k}}$ occurs infinitely many times. However, this is impossible, since $\theta_{k} \rightarrow \theta^{\prime}$ and $\left|f\left(\theta^{\prime}\right)\right| \neq 0$. The contradiction shows $\lim _{k \rightarrow \infty} \lambda_{k}<\infty$.

Case $b\left(\left|f\left(\theta^{\prime}\right)\right|=0\right)$ : The assumption $\lambda_{k} \underset{k \rightarrow \infty}{\longrightarrow} \infty$ implies that there is a sequence of integers $\left\{n_{k}\right\}$ such that $n_{k} \underset{k \rightarrow \infty}{\longrightarrow} \infty$, and $\lambda_{n_{k}+1}=\lambda_{n_{k}}+1$, i.e., for all $k=1,2, \cdots$, the following indicator equals one:

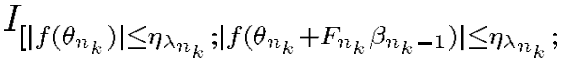

$$
\begin{aligned}
& \left|f\left(\theta_{n_{k}}+F_{n_{k}} \hat{\beta}_{n_{k}}\right)\right| \leq \eta_{\lambda_{n_{k}}} \text {; } \\
& \left.\left|f\left(\theta_{n_{n_{k}}}+\left(1+\sqrt{\eta_{\lambda_{n_{k}}}}\right) F_{n_{n_{k}}} \hat{\beta}_{n_{k_{k}}}\right)\right| \leq \eta_{\lambda_{n_{k}}}\right]=1 \text {. }
\end{aligned}
$$

Take a convergent subsequence of $\left\{\hat{\beta}_{n_{k}}\right\}$. For notational simplicity denote by $\left\{\hat{\beta}_{n_{k}}\right\}$ itself its convergent subsequence. Thus $\hat{\beta}_{n_{k}} \underset{k \rightarrow \infty}{\longrightarrow} \beta^{0}$.
By Theorem 2, $\beta^{0} \in J \backslash\{0\}$.

1) If $\beta^{0} \in J_{2}$, then

$$
f\left(\theta_{n_{k}}+F_{n_{k}} \hat{\beta}_{n_{k}}\right) \rightarrow f\left(\theta^{\prime}+F \beta^{0}\right)=\left\|\beta^{0}\right\|^{l}+b>\eta_{\lambda_{n_{k}}}
$$

for all sufficiently large $k$. Thus, (44) may take place at most a finite number of times. The contradiction shows that $\lim _{k \rightarrow \infty} \lambda_{k}<\infty$.

2) If $\beta^{0} \in J_{1} \backslash\{0\}$, then as $\delta \rightarrow 0$ we have

$$
\begin{aligned}
f\left(\theta^{\prime}+\right. & \left.(1+\sqrt{\delta}) F \beta^{0}\right) \\
& =f\left(\theta^{\prime}+F \beta^{0}\right)+\left(\frac{\partial f\left(\theta^{\prime}+F \beta^{0}\right)}{\partial \beta}\right)^{\tau} \sqrt{\delta} \beta^{0}+O(\delta) \\
& =f\left(\theta^{\prime}+F \beta^{0}\right)+\sqrt{\delta} l\left\|\beta^{0}\right\|^{l-2}\|\beta\|^{2}+O(\delta) .
\end{aligned}
$$

Since $\theta_{k} \rightarrow \theta^{\prime}, F_{k} \rightarrow F, \hat{\beta}_{n_{k}} \rightarrow \beta^{0}$, for sufficiently large $k$ from (45) it follows that

$$
\begin{aligned}
f\left(\theta_{k}\right. & \left.+\left(1+\sqrt{\eta_{\lambda_{n_{k}}}}\right) F_{n_{k}} \hat{\beta}_{n_{k}}\right) \\
& =f\left(\theta_{n_{k}}+F_{n_{k}} \hat{\beta}_{n_{k}}\right)+\sqrt{\eta_{\lambda_{n_{k}}}} l\left\|\hat{\beta}_{n_{k}}\right\|^{l}+O\left(\eta_{\lambda_{n_{k}}}\right)
\end{aligned}
$$

which is greater than $\frac{1}{2} \sqrt{\eta_{\lambda_{n_{k}}}} l\left\|\beta^{0}\right\|^{l}$, if $f\left(\theta_{n_{k}}+\right.$ $\left.F_{n_{k}} \hat{\beta}_{n_{k}}\right) \leq \eta_{\lambda_{n_{k}}}$ and $k$ is large enough. Notice that $\frac{1}{2} \sqrt{\eta_{\lambda_{n_{k}}}} l\left\|\beta^{0}\right\|^{l}>\eta_{\lambda_{r_{k}}}$ if $k$ is large enough. This means that

and

$$
f\left(\theta_{n_{k}}+F_{n_{k}} \hat{\beta}_{n_{k}}\right) \leq \eta_{\lambda_{n_{k}}}
$$

$$
f\left(\theta_{n_{k}}+\left(1+\sqrt{\eta_{\lambda_{n_{k}}}}\right) F_{n_{k}} \hat{\beta}_{n_{k}}\right) \leq \eta_{\lambda_{n_{k}}}
$$

cannot simultaneously happen infinitely many times. This contradicts (43). Thus, $\lim _{k \rightarrow \infty} \lambda_{k}<\infty$ and the proof of the theorem is completed.

\section{CONCLUding REMARKS}

This paper gives the necessary and sufficient condition for (1) to be adaptively stabilizable and presents two methods of parameter modification for the adaptive pole-placement control. One of them is nonrecursive and the other one is recursive, based on stochastic approximation. Both modifications terminate in a finite number of steps and solve the problem.

We note for the case $f\left(\theta^{\prime}\right)=0$, the origin $\beta=0$ is not a stable equilibrium for the equation

$$
\frac{\partial \beta_{t}}{\partial t}=h\left(\beta_{t}\right)
$$

Consequently, the truncation at lower the bound in (28) should be very rare. The computation will be simpler if there is no lower bound truncation.

It is quite remarkable that the adaptive stabilization can be solved without the controllability condition which is necessary for the nonadaptive exact pole-placement problem.

\section{ACKNOWLEDGMENT}

The authors would like to thank Dr. W. P. Li and Prof. $\mathrm{K}$. Yu for helpful discussions concerning algebraic geometry. The authors would also like to thank Prof. L. Guo for useful discussions. 


\section{REFERENCES}

[1] H. F. Chen and J. F. Zhang, "Adaptive stabilization of unstable and nonminimum-phase stochastic systems," Syst. Contr. Lett., vol. 20, pp. 27-38, 1993.

[2] R. Lozano and X. H. Zhao, "Adaptive without excitation probing signals," IEEE Trans. Automat. Contr., vol. 39, pp. 47-58, Jan. 1994

[3] H. F. Chen and J. F. Zhang, "Adaptive pole placement of controllable systems," Sci. China, ser. E, vol. 39, no. 1, pp. 103-112, 1996.

[4] L. Guo, "Self-convergence of weighted least-squares with applications to stochastic adaptive control," IEEE Trans. Automat. Contr., vol. 41, pp. 79-89, Jan. 1996.

[5] B. Bercu, "Weighted estimation and tracking for ARMAX models," SIAM J. Contr. Optimizations, vol. 33, no. 1, pp. 89-106, 1995.

[6] D. Cheng, H. Du, and H. F. Chen, "Geometric structure in stochastic approximation," to be published.

[7] H. F. Chen, T. Duncan, and B. Pasik-Duncan, "On ODE approach to parameter identification and its application to adaptive control," to be published.

[8] H. F. Chen, "Stochastic approximation and its new applications," in Proc. 1994 Hong Kong Int. Wkshp. New Directions Contr. Manufacturing, 1994, pp. 2-12.

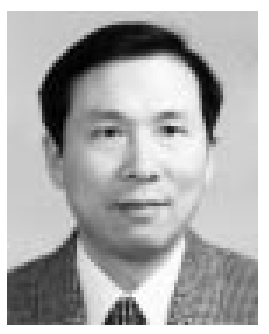

Han-Fu Chen (SM'94-F'97) obtained the Diploma from the Department of Mathematics and Mechanics, Leningrad (St. Petersburg) University, Russia.

$\mathrm{He}$ joined the Institute of Mathematics, Chinese Academy of Sciences, in 1961. Since 1979 he has been with the Institute of Systems Science, Chinese Academy of Sciences. He is a Professor of the Laboratory of System and Control and currently serves as the Director of the Institute. His research interests include stochastic systems, system identification, adaptive control, recursive estimation, and stochastic approximation. He has authored and co-authored more than 120 journal papers.

Mr. Chen is the editor of Systems Science and Mathematical Sciences and is involved in the editorial boards of several international and domestic journals. He was elected to the Chinese Academy of Sciences in 1993. He now serves as the President of the Chinese Association of Automation and is a Standing Member of the Council of the Chinese Mathematics Society. He also serves on the Technical Board of IFAC, chairing the Coordinating Committee "Systems and Signals." For the 14th IFAC Congress to be held in 1999, he serves as the IPC Chairman and NOC Co-Chairman.

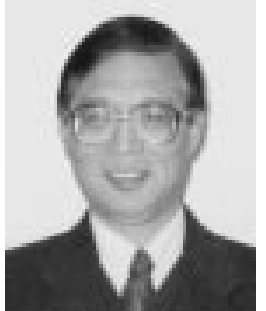

Xi-Ren Cao (S'82-M'84-SM'89-F'96) received the M.S. and Ph.D. degrees from Harvard University, Cambridge, MA, in 1991 and 1984, respectively

He was a Research Fellow at Harvard University from 1984 to 1986 . He then worked as a Principal and Consultant Engineer at Digital Equipment Corporation, USA, until October 1993. Since then, he has been a Professor at the Hong Kong University of Science and Technology. He has published two books: Realization Probabilities-The Dynam ics of Queuing Systems (Springer-Verlag, 1994) and Perturbation Analysis of Discrete-Event Dynamic Systems (Kluwer, 1991). His current research interests include discrete event systems, computer and communication networks, stochastic processes, and optimization techniques.

Dr. Cao received the Outstanding Transactions Paper Award from the IEEE Control System Society in 1987 and the Outstanding Publication Award from the Institution of Management Science in 1990. He served as an Associate Editor of the IEEE TRAnSACTIONS ON Automatic Control and the IEEE Transactions on Control Systems Technology; he is currently an Associate Editor of the Journal of Discrete-Event Dynamic Systems and the chairman of the Technical Committee of Networks and Communications of the IEEE Control Systems Society. 